\title{
Tea Dosage Form Category
}

National Cancer Institute

\section{Source}

National Cancer Institute. Tea Dosage Form Category. NCI Thesaurus. Code C149984.

A type of solid pharmaceutical dose form consisting of one or more herbal drugs

intended for preparation of a solution by decoction or maceration, or a lyophilized

preparation of such a solution. 